\title{
Non-Cardiovascular Computed Tomography Incidental Findings in Patients Who Underwent Transaortic Valve Implantation Procedure
}

\author{
Ahmed Fathala ${ }^{\mathrm{a}, \mathrm{c}}$, Mnahi Bin Saeedan ${ }^{\mathrm{a}}$, Ali Zulfiqar ${ }^{\mathrm{b}}$, \\ Hani Al Sergani ${ }^{b}$
}

\begin{abstract}
Background: Transcatheter aortic valve implantation (TAVI) is a new treatment option for patients with severe aortic stenosis. PreTAVI procedure workup includes computed tomography angiography (CTA) of the heart and aorta from aortic annulus to the iliofemoral arteries. Frequently, there are a number of incidental non-cardiac findings (INCFs) in pre-TAVI CTA. However, the frequency and clinical significance of these INCFs are unknown. The aim of our study was to investigate the prevalence of INCFs and their clinical significance.
\end{abstract}

Methods: This was a retrospective review of 67 patients who underwent dedicated pre-TAVI CTA from 2010 till 2015. Non-cardiovascular INCFs were classified according to their clinical significance into three categories. The first category includes findings that may require urgent treatment. The second category includes findings that need further follow-up. The third category includes incidental findings that require no further follow-up or recommendation.

Results: The total number of patients was 67 , and the mean age was $73 \pm 8$ years. All patients had INCFs and the total number was 248 . Of the patients, $69 \%$ had chest findings, $85 \%$ had abdominal findings, and $33 \%$ had musculoskeletal findings. Results based on categorical classification were as follows: $9 \%, 25 \%$, and $66 \%$ of these 248 findings belong to the first category, the second category, and the third category, respectively.

Conclusion: Non-cardiovascular INCFs are common in pre-TAVI CTA presumably due to increased age of such specific population. These findings have variable clinical significance and some of them might require acute treatment or additional evaluation, and should be managed properly taking into consideration patient's life expectancy and comorbidities.

Manuscript accepted for publication February 02, 2017

${ }^{a}$ Department of Radiology, King Faisal Specialist Hospital \& Research Center, Riyadh, Saudi Arabia

${ }^{b}$ King Faisal Heart Institute, Riyadh, Saudi Arabia

${ }^{\mathrm{c} C o r r e s p o n d i n g ~ A u t h o r: ~ A h m e d ~ F a t h a l a, ~ D e p a r t m e n t ~ o f ~ R a d i o l o g y, ~ K i n g ~ F a i s-~}$ al Specialist Hospital \& Research Center, Riyadh, Saudi Arabia.

Email:ahm35799@hotmail.com
Keywords: TAVI; Cardiac CT; Incidental non-cardiac findings

\section{Introduction}

Aortic stenosis is a common heart disorder that affects nearly $5 \%$ of persons older than 75 years [1]. Many patients with severe aortic stenosis are not surgical candidates for surgical valve replacement owing to associated multiple medical problems and comorbidities [2]. Recently, transaortic valve implantation (TAVI) showed a promise as an alternative option to conventional open heart surgery [3-8]. Multislice detector computed tomography (MDCT) is playing an increasingly important role in patient's screening protocol before TAVI, provides detailed anatomic assessment of aortic valve morphology and calcification, aortic root measurement, aortic annulus size, comprehensive assessment of the aorta and suitability of iliofemoral access, and determines appropriate coaxial angles to optimize the valve implantation procedure $[9,10]$. MDCT images are acquired through the chest, abdomen, and pelvis with a large imaging field of view, and potential incidental non-cardiac findings (INCFs) can be detected. The prior unknown abnormalities that are identified on MDCT without previous suspicion of disease and unrelated to the indications of the examination are referred to as incidental findings [11]. Presence of INCFs at the time of MDCT poses important challenges and management issues. The INCFs may affect the patient's life expectancy following TAVI and overall usefulness of the procedure. Currently, there are limited published data with inconstant results on the prevalence of the INCFs; most published data are small patient's cohort with variable definition and classification of INCFs [12]. The goal of this study was to investigate the prevalence of INCFs in pre-TAVI protocol patients who underwent MDCT as routine investigational workup before TAVI procedure.

\section{Methods}

\section{Study population}

Sixty-seven consecutive patients with severe symptomatic aor- 
Table 1. Patient Characteristic

\begin{tabular}{ll}
\hline Total number of patients (n) & 67 \\
Men (n, \%) & $35(52 \%)$ \\
Women (n, \%) & $32(48 \%)$ \\
Age (years) & $73 \pm 8$ \\
Body mass index $\left(\mathrm{kg} / \mathrm{m}^{2}\right)$ & $31 \pm 7.3$ \\
DM (n, \%) & $52(78 \%)$ \\
HTN (n, \%) & $57(86 \%)$ \\
Hypercholesterolemia $(\mathrm{n}, \%)$ & $35(52 \%)$ \\
History of smoking $(\mathrm{n}, \%)$ & $6(9 \%)$ \\
\hline
\end{tabular}

tic stenosis referred to our institution for pre-TAVI assessment and were retrospectively evaluated between January 2010 and March 2015. The local ethics committee approved the retrospective evaluation of MDCT data of all patients. As a result of the retrospective nature of this study, waiver of informed consent was obtained through the local ethical committee. Mean age was $73 \pm 8$ years, 35 (52\%) patients were male, and $32(48 \%)$ patients were female. Patients with common contraindication to CTA with intravenous contrast such as acute or chronic renal failure or prior contrast reaction were excluded. The study was approved by our local hospital ethics committee.

\section{MDCT protocol}

Patients underwent retrospective ECG gated CT with 64 detectors (HD 750 Discovery; General Electrics, Milwaukee, $\mathrm{WI}$ ), and the CT parameters were as follows: collimation 64 $\times 0.625 \mathrm{~mm}$, section acquisition 0.625 , tube voltage $120 \mathrm{kV}$, tube current based on body mass index, rotation time 330 $\mathrm{ms}$, pitch 0.25 - 0.35 depending on heart rate, scan direction craniocaudal, and iterative reconstruction with $40 \%$ adaptive statistical iterative reconstruction (ASIR) [13]. Contrast materials injection protocol was total dose of material from 90 to $120 \mathrm{~mL}$ of iodixanol 320 (GE, Health Care, Princeton, NJ). The injection rate was $5 \mathrm{~mL} / \mathrm{s}$ followed by $50 \mathrm{~mL}$ of normal saline.

\section{Image analysis}

MDCT images were evaluated by two experienced observers using routine clinical dataset. CT images were reconstructed at slice thickness of $0.75 \mathrm{~mm}$ every $0.5 \mathrm{~mm}$ for coronaries, mediastinal and abdominal images were reconstructed at 2.0 $\mathrm{mm}$ thickness every $1 \mathrm{~mm}$, and images analysis by multiplanar reformation was performed with dedicated software (Cardio Q X-press, General Electrics). The overall quality of the images was good and diagnostic. Images processing datasets included manually measured vessels diameters (aortic root dimensions, aorta, iliac and common femoral arteries), the distance of the coronary ostia to the aortic annulus, and the presence and ex- tent of calcification and touristy [14].

\section{INCFs}

INCFs were diagnosed where an abnormality was found without previous clinical suspicion or known prior disease and were classified into three groups according to the anatomical location. These three groups were chest, abdominal and musculoskeletal. Chest group included lung, mediastinum, airway and thyroid. The abdominal group included gastrointestinal, hepatobiliary, genitourinary and abdominal cavity. The musculoskeletal groups encompassed the bone, joints, muscles and abdominal and chest wall as well as breasts.

Furthermore, on the basis of clinical relevance, non-cardiac findings were classified into three groups. Group 1 findings were clinically significant and needed immediate attention and further evaluation; group 2 findings were clinically significant and needed further follow-up by more imaging with other imaging modalities such as magnetic resonance imaging or ultrasound; group 3 were incidental findings with no further follow-up or recommendations.

\section{Statistical analysis}

Continuous variables with normal distribution were expressed as mean (standard deviation). Variables with skewed distributions were expressed as median. Categorical variables were expressed as frequency (percentage).

\section{Results}

The mean age of the included 67 patients was $73 \pm 8$ years, and 35 male and 32 female (Table 1) showed patient characteristics. At least one INCF was seen in every patient. Forty-six patients $(69 \%)$ had chest findings (Table 2), 57 patients $(85 \%)$ had abdominal findings (Table 3 ) and 22 patients $(33 \%$ ) had musculoskeletal findings (Table 4). The total number of the INCFs in the 67 patients was 248, and number and percentage based on anatomical location were as follows: 95 (38\%) chest findings, 129 (52\%) abdominal and pelvic findings, and $24(10 \%)$ musculoskeletal findings. Of these 248 findings, 23 $(9 \%), 62(25 \%)$, and $153(66 \%)$ findings belong to the first category, the second category, and the third category, respectively (Fig. 1).

Three patients had significant, non-incidental, non-cardiovascular findings including gastric cancer, liver cirrhosis with partly ablated hepatoma and left hip prosthesis loosening, and one patient had malignant course of anomalous left coronary artery.

Distribution of INCFs in category 1 (findings that required treatment and immediate attention) showed most patients ( $83 \%$ ) belong to chest findings, $13 \%$ belong to abdominal findings, and $4 \%$ belong to musculoskeletal findings.

Distribution of INCFs in category 2 showed abdominal findings represent 58\%, 12 patients $(18 \%)$ had liver findings 
Table 2. Distribution and Frequency of the 95 Chest Incidental Non-Cardiac Findings

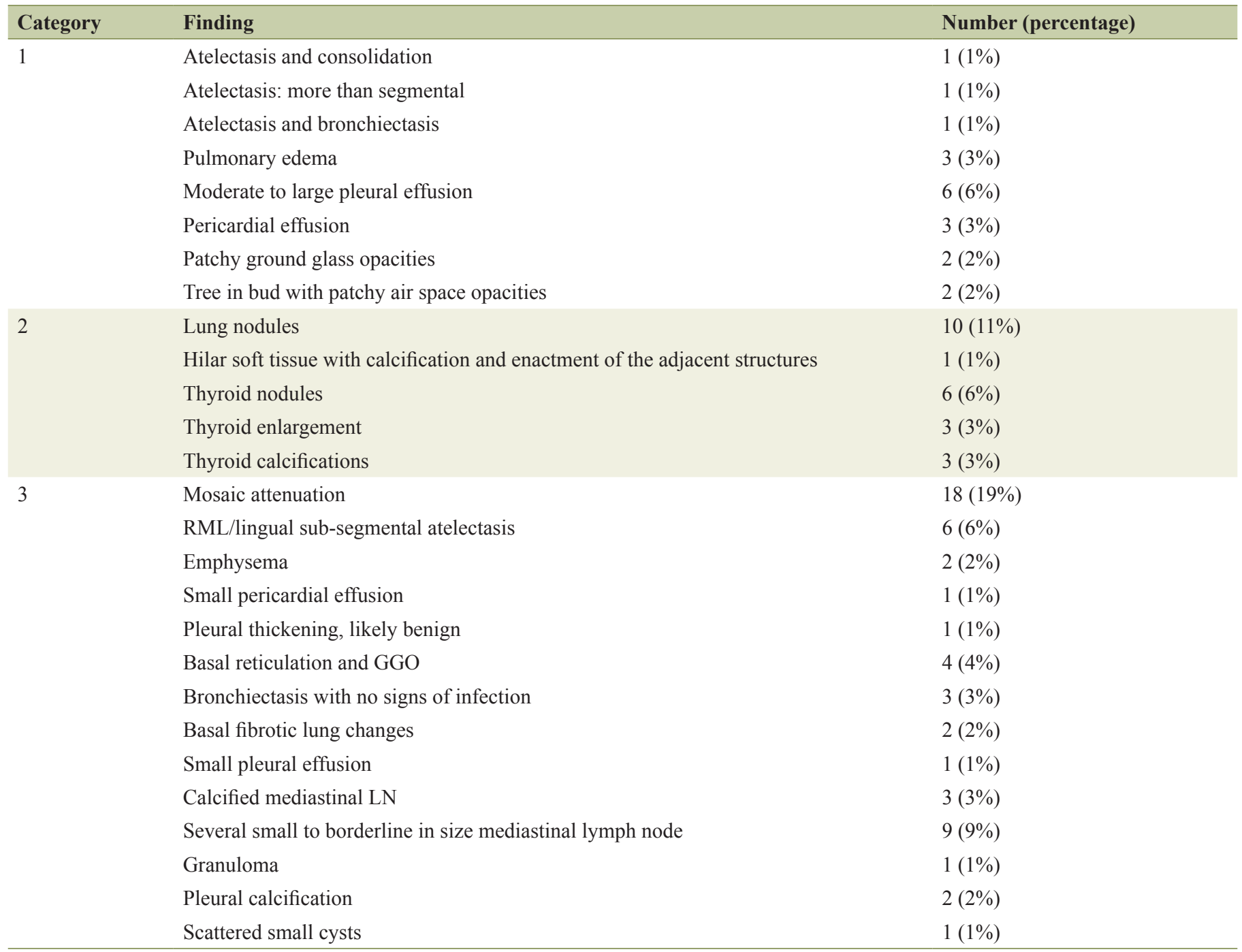

that require further evaluation including solid lesions, one capsular retraction and one liver cirrhosis. Six patients $(9 \%)$ had adrenal gland nodule. Twenty-six patients (39\%) had simple renal cysts belonging to category 3 , while only one patient had cyst with possible internal enhancement requiring further evaluation classified as category 2 . Fifteen patients $(22 \%)$ had heterogeneous prostate enlargement with (seven patients) and without calcifications, chest findings (37\%), and musculoskeletal $(5 \%)$. Ten patients $(15 \%)$ had pulmonary nodules. Twelve patients $(18 \%)$ had thyroid findings including thyroid enlargement (three), nodules (six) and calcifications, and only $3 \%$ had musculoskeletal.

Distribution of INCFs in category 3 showed chest findings represent $53 \%$ of the total chest INCFs, abdominal findings represent $90 \%$ of total abdominal INCFs, and musculoskeletal findings represent $20 \%$ of total musculoskeletal INCFs. Examples of category 3 INCFs include: mosaic lung attenuation, diverticulosis, gallstones, right middle lobe/lingular sub-segmental atelectasis, simple renal cysts, fatty liver, and several small to borderline sized mediastinal lymph nodes.

\section{Discussion}

The main findings in this study are that all patients who underwent pre-TAVI MDCT workup had INCFs. This study is one of few studies that report the prevalence and detailed nature of the non-cardiovascular findings in such specific population. This is the only study, to the best of our knowledge, that addresses the incidental findings based on anatomical location such as chest, abdominal and musculoskeletal findings. This system-based approach will be of great assist to interpreting physician to look for and report these findings more precisely in a systematic method. A more conservative approach in the classification of incidental findings was followed; particularly in category 1 , there are findings that we think that need to be managed more promptly, such as anterior abdominal wall fat stranding and early abscess formation, and pulmonary edema 
Table 3. Distribution and Frequency of the 129 Abdominal Incidental Non-Cardiac Findings

\begin{tabular}{|c|c|c|}
\hline Category & Finding & Number (percentage) \\
\hline 1 & Moderate amount of ascites & $3(2 \%)$ \\
\hline \multirow[t]{10}{*}{2} & Prostate enlargement (seven with calcification) & $15(12 \%)$ \\
\hline & Adrenal nodule & $4(5 \%)$ \\
\hline & Hypodense liver lesion & $4(5 \%)$ \\
\hline & Hypervascular liver lesion & $3(2 \%)$ \\
\hline & Liver capsular retraction and hypodensity & $1(1 \%)$ \\
\hline & Features of liver cirrhosis & $1(1 \%)$ \\
\hline & Cystic pancreatic lesion & $1(1 \%)$ \\
\hline & Renal cyst with possible internal enhancement & $1(1 \%)$ \\
\hline & Persplenic partly calcified soft tissue mass & $1(1 \%)$ \\
\hline & Fluid filled heterogeneous uterus & $1(1 \%)$ \\
\hline \multirow[t]{20}{*}{3} & Diverticulosis & $14(11 \%)$ \\
\hline & Gallstones & $4(5 \%)$ \\
\hline & Splenomegaly with large SV and PV & $1(1 \%)$ \\
\hline & Hiatal hernia & $3(2 \%)$ \\
\hline & Duodenal lipoma & $1(1 \%)$ \\
\hline & Dilated esophagus & $1(1 \%)$ \\
\hline & Absence left kidney & $1(1 \%)$ \\
\hline & Renal stone & $1(1 \%)$ \\
\hline & Simple renal cyst & $26(20 \%)$ \\
\hline & Renal scaring & $2(2 \%)$ \\
\hline & Renal atrophy & $5(4 \%)$ \\
\hline & Renal infarction & $2(2 \%)$ \\
\hline & Fibroid & $6(5 \%)$ \\
\hline & Fatty liver & $14(11 \%)$ \\
\hline & Calcified abdominal lymph nodes & $1(1 \%)$ \\
\hline & Splenic infraction & $2(2 \%)$ \\
\hline & Liver cyst & $1(1 \%)$ \\
\hline & Peripheral splenic calcification & $1(1 \%)$ \\
\hline & Transient intussusception & $1(1 \%)$ \\
\hline & Gastric diverticulum & $1(1 \%)$ \\
\hline
\end{tabular}

and presence of lung infection, e.g., tree in bud appearance and patchy ground glass opacity. Early identification and prompt management of such abnormality may have a major impact in patients' management and long-term outcome.

A total of 248 INCFs were identified in our study population. The most common INCF belongs to abdominal category; a similar study showed that $99 \%$ of 200 patients had 456 of non-INCFs, and most patients had multiple findings that fell into more than one group. Incidental abdominal and pelvic findings contributed to 129 (52\%) of the total findings (Table 3 ); interestingly, few patients belong to category 1 , such as moderate to severe ascites, but majority of findings in category 2 that need further follow-up, and group 3 findings that incidental and further workup or recommendation was required.
As per Table 3, there are multiple findings in category 2 including liver lesions, adrenal nodules, pancreatic and renal lesion, and presence of these incidental findings obviously, as per standard practice, initiates more workup. However, these findings are not contraindication to TAVI procedure.

Chest INCFs compose 95 (38\%) of total findings, findings that need urgent management are more common than abdominal findings, and these findings include lung consolidation, atelectasis, pulmonary edema, pericardial effusion, and evidence of ongoing lung infection such as ground glass opacities and tree in bud. Prompt identification and management of these incidental findings is of a great clinical value to prevent further clinical deterioration and to avoid short- and long-term complication. Only lung nodules, hilar and mediastinal abnormali- 
Table 4. Distribution and Frequency of the 24 Musculoskeletal Incidental Non-Cardiac Findings

\begin{tabular}{lll}
\hline Category & Finding & Number (percentage) \\
\hline 1 & Anterior abdominal wall thickening, fat stranding with foci of air, query cellulitis & $1(4 \%)$ \\
2 & Osteopenia with non-aggressive iliac lytic areas & $1(4 \%)$ \\
Lumbar vertebrae complete collapse with posterior bulge & $1(4 \%)$ \\
Non-aggressive well defined lytic iliac bone lesion with internal calcification & $1(4 \%)$ \\
3 & Spondololysis & $3(13 \%)$ \\
Sacroilitots & $1(4 \%)$ & $1(4 \%)$ \\
DISH & Spine compression fracture & $2(8 \%)$ \\
Elastofibroma dorsi & $1(4 \%)$ \\
Non-complicated abdominal wall hernia & $5(21 \%)$ \\
Gynecomastia & $2(8 \%)$ \\
Muscle lipoma & $2(8 \%)$ \\
Breast calcification & $1(4 \%)$ \\
Calcified nodes in the axillary region and pelvic muscles & $1(4 \%)$ \\
\end{tabular}

ties, and thyroid nodules in category 2 need further follow-up; on the other hand, there are multiple incidental chest findings that do not need any further workup (Table 2).

Musculoskeletal findings are the smallest group, only 24 $(10 \%)$, only one finding, anterior abdominal wall thickening, foci of air, and fat stranding that we thought it needs immediate attention. Findings required follow-up such as vertebral collapse with possible cord compression and non-aggressive bone lesions. However, the majorities of MSK findings are incidental and require no further workup such as non-complicated abdominal wall hernia, lipoma, mild scoliosis, and calcified nodes in the axillary and pelvic muscle (Table 4).

The first category findings are likely related to patient's se- vere aortic stenosis. This includes moderate amount of ascites in three patients, moderate to large amount pleural effusion in six patients, moderate to large amount pericardial effusion in three patients and pulmonary edema in three patients, and anterior abdominal wall infection in MSK findings.

The second category findings are more interesting as they might require further evaluation or correlation with additional radiological or non-radiological tests. Ten $(15 \%)$ patients had pulmonary nodules. Lung nodule is a common incidental finding with the new generation chest CT examinations and has been reported in up to $51 \%$ of smokers aged 50 years or older. Fleischner's society recommendations for follow-up and management of lung nodules smaller than $8 \mathrm{~mm}$ detected inciden-

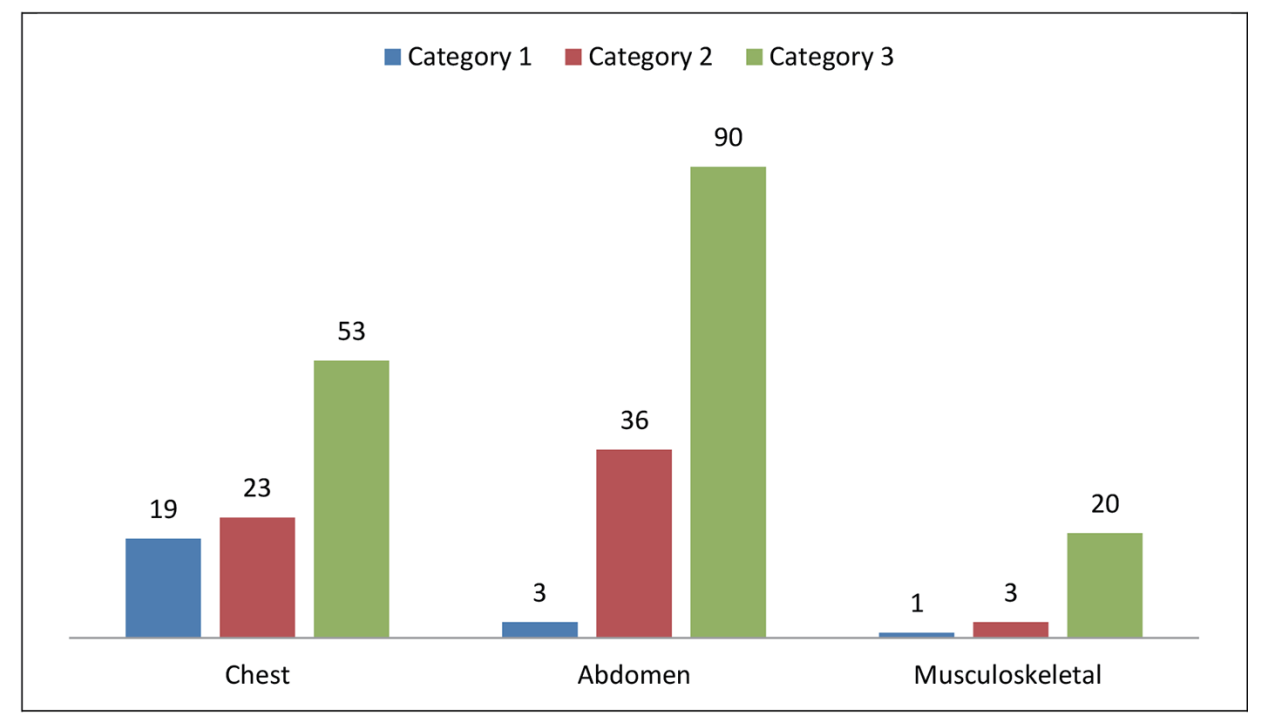

Figure 1. Number and distribution of 248 non-cardiovascular incidental findings in 67 patients by system and categories. 
tally at non-screening CT in persons 35 years of age or older depend on the nodule size (average of length and width) and individual's risk factor of lung cancer like smoking. Twelve patients $(18 \%)$ had thyroid findings including thyroid enlargement, nodules, and calcifications. Incidental thyroid nodules (ITNs) are common. Further evaluation of ITN including neck ultrasound and possibly FNA should take into consideration nodule size, imaging features and the patient's life expectancy, based primarily on age and comorbidities. Only one patient had a cyst with possible internal enhancement requiring further evaluation classified as category Bosniak two. Incidental renal findings are common and mostly cystic in nature and classified as Bosniak category 1 . Twelve patients (18\%) had liver findings that require further evaluation including solid lesions, one capsular retraction and one liver cirrhosis. Incidental liver findings should be managed based on the individual risk stratification. Low (age $<40$ years) and average (age $>40$ years) risk individuals have no known 1ry malignancies with a propensity to metastasize to the liver, cirrhosis, or other hepatic risk factors. Further evaluation is not required for such patients with sharply marginated solitary or multiple masses with low-attenuation (20 HU). Six patients $(9 \%)$ had adrenal gland nodule. Adrenal nodule is a relatively common finding. Lesion size, density features, stability as compared to prior if available, presence or absence of 1ry malignancy are important factors that lead to the proper management. Presence of macroscopic fat with or without calcifications is diagnostic of benign adrenal myelolipoma. Homogenous adrenal nodule with HU less than 10 in non-enhanced CT scan is most likely fat rich adenoma. However, TAVI dedicated CT scans are aortic angiography which might interfere with this role. Adrenal lesion of $1-4 \mathrm{~cm}$ size and benign features (low density, homogeneous, smooth margins) with no prior imaging or history of 1ry malignancy could be followed up after 1 year with CT or MRI.

The third category findings are more common, and typically these finding are incidental and require no further workup. For abdominal findings such as diverticulosis, gallstones, and fatty liver, benign renal cysts are very common in this age group. Common chest incidental findings such as granuloma scatter small cyst, and small pleural effusion and small calcified mediastinal lymph nodes, particularly have no major clinical relevance.

Our major study findings are consistent with previous studies. Lindsay et al in a recent study demonstrated that incidental findings in pre-TAVI CT are common, and do not independently identify patients with poor outcomes after TAVI procedure [11]. Detection of incidental findings on CT should not necessarily influence or delay the decision to perform TAVI. In a total number of 260 patients INCFs were observed in 204 patients $(70.6 \%)$, eight $(3.9 \%)$ malignances were detected, and the authors concluded a high prevalence of noncardiac findings on routine pre-TAVI CT examination [12]. In another study by Gufler et al, in a total of 131 patients, $31 \%$ patients had significant non-cardiac findings, 19\% chest and $12 \%$ abdominal findings. Five lesions were considered potentially malignant, two renal cell carcinomas, one hepatoma, one mediastinal lymphoma, and one metastatic bladder cancer. The study found that prevalence of malignant incidental finding in
pre-TAV CT is $3.8 \%$. However, this prevalence is not high in TAVI patients with average age of 81.6 years compared to general population [15]. A 2-year survival after TAVI procedure was investigated by Stachon et al, and the presence of potentially malignant findings (PMF) versus no PMF did not significantly influence decision or time to treatment. Several findings which are highly suspicious for malignancy were less likely associated with invasive treatment; the authors concluded that the frequently occurring radiological PMF did not influence 2-year survival after TAVI procedure [16].

\section{Limitations}

There are some important limitations. This is a single-center study with relatively small number of patients, which might have some invalid representations of the population; however, compared to other similar studies, our patients comparable to others, typical TAVI patients are elderly with multiple associated commodities. One of the other important limitations is no follow-up of most of the patients, the real significance and impact of non-cardiac findings are unknown, but all our patients underwent successful TAVI procedure and discharged home without any in-hospital complications, and most of the patients as per radiological findings will follow with their primary care physicians.

\section{Conclusion}

INCFs are common in routine pre-TAVI CT presumably due to advanced age of patient population. These findings have variable clinical significance and some of them might require acute treatment while other might require additional evaluation and follow-up. Most INCFs do not influence the decision to perform TAVI procedure, and do not affect post-procedure outcome. Heart team should be aware of this burden of potentially significant INCF. It is imperative to report pre-TAVI CT; beside cardiac and vascular findings, such incidental findings must be reported in a systemic method in a system-based approach to avoid missing important findings.

\section{References}

1. Nkomo VT, Gardin JM, Skelton TN, Gottdiener JS, Scott CG, Enriquez-Sarano M. Burden of valvular heart diseases: a population-based study. Lancet. 2006;368(9540):1005-1011.

2. Iung B, Baron G, Butchart EG, Delahaye F, Gohlke-Barwolf C, Levang OW, Tornos $\mathrm{P}$, et al. A prospective survey of patients with valvular heart disease in Europe: The Euro Heart Survey on Valvular Heart Disease. Eur Heart J. 2003;24(13):1231-1243.

3. Smith CR, Leon MB, Mack MJ, Miller DC, Moses JW, Svensson LG, Tuzcu EM, et al. Transcatheter versus surgical aortic-valve replacement in high-risk patients. $\mathrm{N}$ Engl J Med. 2011;364(23):2187-2198.

4. Leon MB, Smith CR, Mack M, Miller DC, Moses JW, 
Svensson LG, Tuzcu EM, et al. Transcatheter aortic-valve implantation for aortic stenosis in patients who cannot undergo surgery. N Engl J Med. 2010;363(17):1597-1607.

5. Lichtenstein SV, Cheung A, Ye J, Thompson CR, Carere RG, Pasupati S, Webb JG. Transapical transcatheter aortic valve implantation in humans: initial clinical experience. Circulation. 2006;114(6):591-596.

6. Walther T, Falk V, Borger MA, Dewey T, Wimmer-Greinecker G, Schuler G, Mack M, et al. Minimally invasive transapical beating heart aortic valve implantation - proof of concept. Eur J Cardiothorac Surg. 2007;31(1):9-15.

7. Walther T, Simon P, Dewey T, Wimmer-Greinecker G, Falk V, Kasimir MT, Doss M, et al. Transapical minimally invasive aortic valve implantation: multicenter experience. Circulation. 2007;116(11 Suppl):I240-245.

8. Bruschi G, De Marco F, Fratto P, Oreglia J, Colombo P, Botta L, Klugmann S, et al. Alternative approaches for trans-catheter self-expanding aortic bioprosthetic valves implantation: single-center experience. Eur J Cardiothorac Surg. 2011;39(6):e151-158.

9. Tops LF, Wood DA, Delgado V, Schuijf JD, Mayo JR, Pasupati S, Lamers FP, et al. Noninvasive evaluation of the aortic root with multislice computed tomography implications for transcatheter aortic valve replacement. JACC Cardiovasc Imaging. 2008;1(3):321-330.

10. Schultz CJ, Moelker AD, Tzikas A, Rossi A, van Geuns RJ, de Feyter PJ, Serruys PW. Cardiac CT: necessary for precise sizing for transcatheter aortic implantation. EuroIntervention. 2010;6(Suppl G):G6-G13.

11. Lindsay AC, Sriharan M, Lazoura O, Sau A, Roughton
M, Jabbour RJ, Di Mario C, et al. Clinical and economic consequences of non-cardiac incidental findings detected on cardiovascular computed tomography performed prior to transcatheter aortic valve implantation (TAVI). Int $\mathrm{J}$ Cardiovasc Imaging. 2015;31(7):1435-1446.

12. Staab W, Bergau L, Lotz J, Sohns C. Prevalence of noncardiac findings in computed tomography angiography before transcatheter aortic valve replacement. J Cardiovasc Comput Tomogr. 2014;8(3):222-229.

13. Gurvitch R, Wood DA, Leipsic J, Tay E, Johnson M, Ye J, Nietlispach F, et al. Multislice computed tomography for prediction of optimal angiographic deployment projections during transcatheter aortic valve implantation. JACC Cardiovasc Interv. 2010;3(11):1157-1165.

14. Achenbach S, Delgado V, Hausleiter J, Schoenhagen P, Min JK, Leipsic JA. SCCT expert consensus document on computed tomography imaging before transcatheter aortic valve implantation (TAVI)/transcatheter aortic valve replacement (TAVR). J Cardiovasc Comput Tomogr. 2012;6(6):366-380.

15. Gufler H, Schulze CG, Wagner S. Incidental findings in computed tomographic angiography for planning percutaneous aortic valve replacement: advanced age, increased cancer prevalence? Acta Radiol. 2014;55(4):420-426.

16. Stachon P, Kaier K, Milde S, Pache G, Sorg S, Siepe M, von zur Muhlen C, et al. Two-year survival of patients screened for transcatheter aortic valve replacement with potentially malignant incidental findings in initial body computed tomography. Eur Heart J Cardiovasc Imaging. 2015;16(7):731-737. 